\title{
Vortex Structure in Superconducting Iron Pnictide Single Crystals
}

\author{
L. Ya. Vinnikov ${ }^{a, *}$, T. M. Artemova ${ }^{a, b}$, I. S. Veshchunov ${ }^{a}$, N. D. Zhigadlo ${ }^{c}, J^{\prime}$ Karpinskic ${ }^{c}$, \\ P. Popovich ${ }^{d}$, D. L. Sun ${ }^{d}$, C. T. Lin ${ }^{d}$, and A. V. Boris ${ }^{d, e}$ \\ ${ }^{a}$ Institute of Solid State Physics, Russian Academy of Sciences, Chernogolovka, Moscow region, 142432 Russia \\ *e-mail: vinnik@issp.ac.ru \\ ${ }^{b}$ Moscow Institute of Physics and Technology, Institutskiu per. 9, Dolgoprudny̆, Moscow region, 141700 Russia \\ ${ }^{c}$ Laboratory for Solid State Physics, Eidgenössische Technische Hochschule Zürich, Zürich, 8093 Switzerland \\ ${ }^{d}$ Max Planck Institute for Solid State Research, D-70569 Stuttgart, Germany \\ ${ }^{e}$ Department of Physics, Loughborough University, Loughborough, LE11 3TU, United Kingdom
}

Received July 14, 2009

\begin{abstract}
The vortex structure in the iron pnictide single crystals $\mathrm{Ba}_{1-x} \mathrm{~K}_{x} \mathrm{Fe}_{2} \mathrm{As}_{2}$ and $\mathrm{Sr}_{1-x} \mathrm{~K}_{x} \mathrm{Fe}_{2} \mathrm{As}_{2}$ of the 122 type and $\mathrm{SmFeAsO}_{1-x} \mathrm{~F}_{x}$ of the 1111 type has been investigated using the decoration method. In all of the crystals under investigation, no regular vortex lattice has been revealed in the magnetic field range up to 200 Oe. The disordered vortex structure is discussed in view of the vortex pinning in single crystals.
\end{abstract}

PACS numbers: 74.25.Qt, 74.70.-b, 74.72-h

DOI: $10.1134 / \mathrm{S} 0021364009160152$

Interest in superconducting layered iron-based compounds as a new class of high-temperature superconductors was manifested in reviews [1-4], where they are compared to cuprate superconductors [2, 3]. As well as cuprates, superconducting pnictides are layered compounds where the $\mathrm{Fe}-\mathrm{As}$ layer is conditionally considered as "superconducting," while the remaining components in the separating layers supply the carriers to the $\mathrm{Fe}-\mathrm{As}$ layer. However, anisotropy in these compounds is insufficiently large [5] to classify them as two-dimensional compounds, as the BSCCO family, and they are closer to the YBCO family in this parameter. Moreover, the anisotropy degree of the superconducting iron pnictides decreases as the oxygen-containing $\mathrm{R}\left(\mathrm{O}_{1-x} \mathrm{~F}_{x}\right)$ separating layers $(\mathrm{R}=\mathrm{La}$, $\mathrm{Sm}, \mathrm{Nd}, \ldots)$ are replaced by the monoatomic $\left(\mathrm{A}_{1-x} \mathrm{~B}_{x}\right)$ layers $(\mathrm{A}=\mathrm{Ba}, \mathrm{Sr}, \mathrm{Ca}, \mathrm{B}=\mathrm{K}, \mathrm{Cs}, \mathrm{Na})$. In this case, the crystal structure of the 1111$\mathrm{RFeAs}\left(\mathrm{O}_{1-x} \mathrm{~F}_{x}\right)$ family corresponds to the tetragonal symmetry and the space group $P 4 / \mathrm{nmm}$ ( $\mathrm{ZrCuSiAs}$ type) and the $122-\mathrm{A}_{1-x} \mathrm{~B}_{x} \mathrm{Fe}_{2} \mathrm{As}_{2}$ family has the space group I4/mmm (a $\mathrm{ThCr}_{2} \mathrm{Si}_{2}$-type lattice). The initial "parent" compounds of the 122 and 1111 type are antiferromagnetic metals, unlike cuprates [2]. The substitution in the separating layers results in the electron and hole doping of the Fe-As layers in the 1111 and 122 compounds, respectively, thus suppressing antiferromagnetism and enhancing superconductivity [1-4]. Superconductivity also arises in both structures under electron doping via the direct substitution of Co or $\mathrm{Ni}$ for $\mathrm{Fe}$ positions in the $\mathrm{Fe}-\mathrm{As}$ layers. The distinc- tive features mentioned in [2] can be supplemented by unusually high critical currents $J_{c}$, i.e., strong pinning in the single crystals [6]. For example, $J_{\mathrm{c}}=10^{6} \mathrm{~A} / \mathrm{cm}^{2}$ in the $\mathrm{SmFeAsO}_{1-x} \mathrm{~F}_{x}$ single crystals in a magnetic field of $60 \mathrm{kG}$ [7]. Communications [6, 7] on high critical currents in single crystals based on iron compounds and the observation of the disordered vortex structure in $\mathrm{Ba}\left(\mathrm{Fe}_{0.93} \mathrm{Co}_{0.07}\right)_{2} \mathrm{As}_{2}$ single crystals over a wide range of magnetic fields from units of millitesla to $9 \mathrm{~T}$ performed by the three known independent methods (decoration, neutron diffraction [8], and scanning tunneling microscopy [9]) suggest the possibility of an unusual mechanism of vortex pinning in pnictides. It is known that the strong pinning is attributed to crystal irregularities of the order of the superconductor coherence length $\xi[10,11]$. The question arises as to whether this irregularity is properly associated with the complicated electron structure of pnictides, which are multiband and affected by the $\mathrm{Fe}-3 d$ magnetic moment, or it is improper and depends on the crystal growth conditions and the quality of the crystals. In particular, one of the probable causes of the strong pinning observed in $\mathrm{Ba}\left(\mathrm{Fe}_{0.93} \mathrm{Co}_{0.07}\right){ }_{2} \mathrm{As}_{2}[8,9]$ could be the crystal structure faults caused by the Co substitution directly in the $\mathrm{Fe}$-As superconducting planes. In this respect, it is interesting to study the vortex structure in the $\mathrm{RFeAs}\left(\mathrm{O}_{1-x} \mathrm{~F}_{x}\right)$ and $\mathrm{A}_{1-x} \mathrm{~B}_{x} \mathrm{Fe}_{2} \mathrm{As}_{2}$ crystals where the doping of the $\mathrm{Fe}-\mathrm{As}$ planes does not disturb their structure. Moreover, the anomalous vortex pinning could be caused by the phase separation with the inclusion of the second phase of size $\xi$. 
Table

\begin{tabular}{l|l|c|c}
\hline \multicolumn{1}{c|}{ Sample } & \multicolumn{1}{|c|}{ Formula } & $T_{\mathrm{c}}, \mathrm{K}$ & $\lambda *, \mu \mathrm{m}$ \\
\hline Ba122 no. 1 & $\mathrm{Ba}_{0.8} \mathrm{~K}_{0.2} \mathrm{Fe}_{2} \mathrm{As}_{2}$ & 30 & $0.28 \pm 0.03$ \\
Ba122 no. 2 & $\mathrm{Ba}_{0.65} \mathrm{~K}_{0.35} \mathrm{Fe}_{2} \mathrm{As}_{2}$ & 38.5 & $0.2 \pm 0.02$ \\
Ba122 no. 3 & $\mathrm{Ba}_{0.6} \mathrm{~K}_{0.4} \mathrm{Fe}_{2} \mathrm{As}_{2}$ & 25 & - \\
Sm1111 & $\mathrm{SmFeAsO}_{1-x} \mathrm{~F}_{x}$ & $45-53$ & $0.2 \pm 0.05$ \\
Sr122 & $\mathrm{Sr}_{0.85} \mathrm{~K}_{0.15} \mathrm{Fe}_{2} \mathrm{As}_{2}$ & 32 & $0.18 \pm 0.03$ \\
BSCCO & $\mathrm{Bi}_{2} \mathrm{Sr}_{2} \mathrm{CaCu}_{2} \mathrm{O}_{8-x}$ & 84 & $0.2 \pm 0.03$ \\
\hline
\end{tabular}

Indeed, the phase separation in the undoped $\mathrm{Ba}_{1-x} \mathrm{~K}_{x} \mathrm{Fe}_{2} \mathrm{As}_{2}$ crystals has been indicated in recent studies by the muon spin relaxation $(\mu \mathrm{SR})[12]$ and angular resolution photoelectron spectroscopy (ARPES) [13]. However, this phase separation could be expected to strongly depend on the doping degree.

In this work, the vortex structure of the single crystals of the electron-doped $\mathrm{SmFeAsO}_{1-x} \mathrm{~F}_{x}(\mathrm{Sm}-1111)$ and hole-doped $\mathrm{Ba}(\mathrm{Sr})_{1-x} \mathrm{~K}_{x} \mathrm{Fe}_{2} \mathrm{As}_{2}$ (Ba(Sr)-122) superconductors with different doping degrees is investigated by the decoration method [14]. The $\mathrm{Ba}(\mathrm{Sr})_{1-x} \mathrm{~K}_{x} \mathrm{Fe}_{2} \mathrm{As}_{2}$ single crystals were grown at the Max Planck Institute of Solid State Research (Stuttgart, Germany) in a nitrogen-filled sealed quartz ampoule with or without the tin flux. The excessive K content of $30 \mathrm{wt} \%$ was added to the burden to compensate losses at a high melting temperature. The cooling was performed at a rate of $3{ }^{\circ} \mathrm{C}$ per hour down to $550^{\circ} \mathrm{C}$. The grown crystals were removed from the flux. The single crystal growth method, compositions, and crystal structures were earlier described in [15]. The chemical composition of the single crystals under study was controlled by local X-ray spectral analysis, and the superconducting transition temperature $T_{\mathrm{c}}$, by the resistivity measurements or the magnetic susceptibility measurements. We note that the Ba122 no. 1 and no. 3 and Sr122 single crystals (see table) were grown in the tin flux, while the optimally doped Ba122 no. 2 single crystals with the maximum transition temperature $T_{\mathrm{c}}=38.5 \mathrm{~K}$ were self-flux grown. The single crystals of the $1111 \mathrm{SmFeAsO}_{1-x} \mathrm{~F}_{x}$ family were grown at Eidgenössische Technische Hochschule (Zürich, Switzerland). The details of the single crystal synthesis were presented in [7]. It is rather difficult to synthesize the 1111-type single crystals, and their samples obtained presently by high-pressure synthesis are of a very small size no greater than $250 \mu \mathrm{m}[7,16]$.

The vortex structure was investigated on the single crystals with an optically smooth (lustrous) surface on the basis plane both as-grown and finished by peeling the surface layer with an adhesive tape. The decoration was performed in the field cooling mode in a magnetic field normal to the basis plane (parallel to the $c$ axis) in the range of $10-200 \mathrm{Oe}$ at $\sim 5 \mathrm{~K}$. In every experiment, the BSCCO single crystals were used as reference samples, which were placed in the decoration chamber near the pnictide single crystals under study. In the investigation of the vortex structure in the same sample in different magnetic fields, the magnetic particles of the previous decoration process were removed by rinsing with isopropyl alcohol or by removing the surface layer with adhesive tape.

Figures 1a, 1c, and 1d illustrate the absence of any order in the vortex arrangement in the same optimally doped Ba122 no. 2 single crystal (see table) throughout the magnetic field range under investigation, while a regular triangular vortex lattice was observed in the reference BSCCO single crystal even at the lowest magnetic fields (see Fig. 1b). This pattern for the B122 no. 2 single crystal was conserved up to a magnetic field of $200 \mathrm{Oe}$; at higher magnetic fields, isolated vortices are poorly resolved, although the vortex lattice was clearly observed for BSCCO. A similar structure was also observed for the Ba122 no. 1 and no. 3 single crystals, but it was observed for the Ba122 no. 1 single crystal only up to a magnetic field of $\sim 80$ Oe. Figure $2 a$ presents the vortex structure on the Sm1111 single crystal where no regular arrangement of the vortices is observed. Owing to the small size of the samples and the difficulties in their attachment to the substrate, we could not investigate their vortex structure in various magnetic fields. The vortex structure in the Sr122 single crystals was only observed in separate surface regions (see Fig. 2b).

In all of the samples investigated where the vortex structure was well resolved, the estimate of a magnetic flux quantum coincided with the theoretical one, $2 \times$ $10^{-7} \mathrm{G} / \mathrm{cm}^{2}$. In low magnetic fields when separate vortices are well resolved, the visible "diameter" of the vortex image can serve as the upper estimate of the London magnetic field penetration depth $\lambda^{*}$ [17]. This estimate obtained from the measurements in the fields lower than $30 \mathrm{Oe}$ is $\lambda^{*}=0.21 \pm 0.03 \mu \mathrm{m}$ for all of the samples under study, except for Ba122 no. 1 for which $\lambda^{*}=0.28 \pm 0.03 \mu \mathrm{m}$. A considerable increase in the penetration depth for the Ba122 no. 1 single crystals explains the impossibility of resolving the vortex structure in magnetic fields above $80 \mathrm{Oe}$. The same data, at the known Ginzburg-Landau parameter value, can provide a lower estimate for the first critical field. Although the accuracy of these measurements is low, these estimates are quite reasonable in view of the difficulty of the experimental determination of the absolute values of $\lambda$ and $H_{\mathrm{cl}}$.

The main result of the observation of the vortex structure in all of the Fe-containing pnictide single crystals studied in this work is the absence of the vortex lattice. We note that the regular vortex lattice was always observed in all of the BSCCO reference samples in every experiment. In combination with the data on high critical currents in the $\mathrm{Ba}\left(\mathrm{Fe}_{0.93} \mathrm{Co}_{0.07}\right)_{2} \mathrm{As}_{2}$ $\left(J_{\mathrm{c}}=10^{5} \mathrm{~A} / \mathrm{cm}^{2}[6]\right)$ and $\operatorname{Sm} 1111\left(J_{\mathrm{c}}=10^{6} \mathrm{~A} / \mathrm{cm}^{2}\right.$ at 

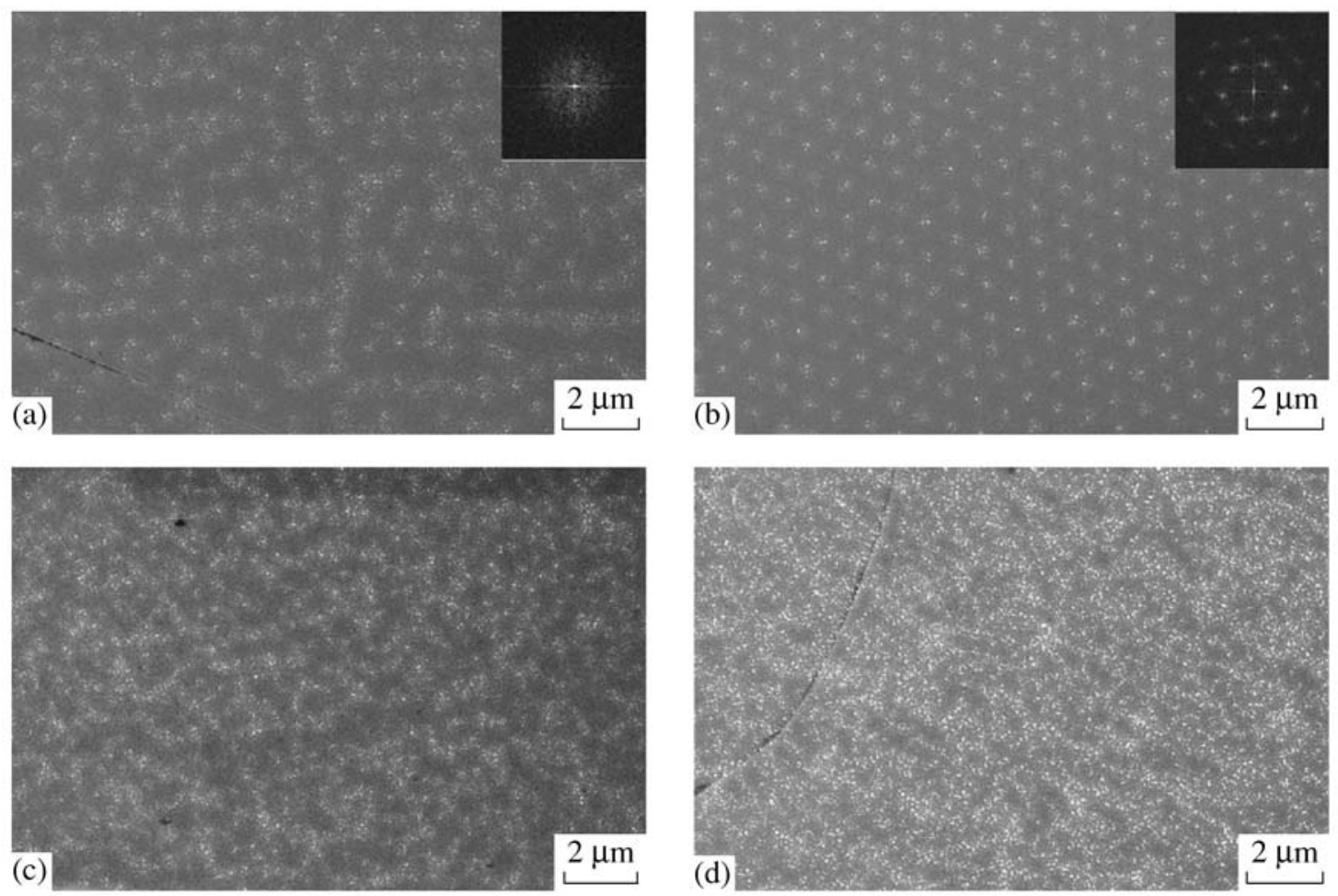

Fig. 1. Vortex structure in the (a) $\mathrm{Ba}_{0.65} \mathrm{~K}_{0.35} \mathrm{Fe}_{2} \mathrm{As}_{2}\left(T_{\mathrm{c}}=38.5 \mathrm{~K}\right.$ ) and (b) BSCCO-2212 single crystals in a magnetic field of $30 \mathrm{Oe}$, as well as in $\mathrm{Ba}_{0.65} \mathrm{~K}_{0.35} \mathrm{Fe}_{2} \mathrm{As}_{2}$ in magnetic fields of (c) 80 and (d) 160 Oe. The insets show the Fourier patterns in an arbitrary scale the same for panels (a) and (b).

$5 \mathrm{~K}$ [7]) single crystals studied, our results point to strong pinning [8].

The cause of the strong pinning in the iron pnictide single crystals has not yet been revealed. The effective pinning centers could be the Co nanoparticles that were observed in the $\mathrm{Ba}\left(\mathrm{Fe}_{x} \mathrm{Co}_{1-x}\right)_{2} \mathrm{As}_{2}$ single crystals using a scanning tunneling microscope, but no correlation in the distributions of vortices and these particles was observed [9]. The probable cause of the disordering of the vortex structure in the pnictide single crystals could be the electron phase separation. Indeed, we investigated the underdoped single crystals Ba122 no. 1 of the series of samples where the coexistence of the superconducting and magnetic phases has been experimentally confirmed $[12,13]$. However, no qualitative difference between the vortex structures of samples Ba122 no. 1, Ba122 no. 2, and Ba122 no. 3 with different doping levels was observed. Moreover, according to the estimate from the low-temperature measurements of the specific heat, the volume of the superconducting phase in Ba122 no. 2 is no less than $98.5 \%$, and this points to the absence of phase separation in these samples. Nonetheless, the vortex lattice is absent (see Figs. 1a, 1c, 1d).

These peculiarities were not observed in the other multicomponent systems, for example, in the twinfree YBCO (124) [18] or BSCCO (2212) [19], where the vortex lattices were clearly seen in the single crys- tals, and the critical currents were several orders of magnitude lower. Meanwhile, a regular vortex lattice [17] was observed in the $\mathrm{MgB}_{2}$ single crystals, which have synthesis conditions close to those of the Sm1111 compound [7]. The common structural component of all of the single crystals studied is the "superconducting" $\mathrm{Fe}-\mathrm{As}$ layer. For the 122-type crystals in the $\mathrm{Ba}\left(\mathrm{Fe}_{0.93} \mathrm{Co}_{0.07}\right)_{2} \mathrm{As}_{2}$ compounds, the substitution of Co for Fe can distort the structure of the superconducting $\mathrm{Fe}$-As layer itself, while the doping of the 122 family with potassium is accompanied by the introduction of distortions into the nonsuperconducting layer. Since the doping leads to nonstoichiometry and possible spatial distortions on the atomic scale shorter than the coherence length, it cannot lead to the strong pinning of the vortices, as observed in the case of the HTSC cuprates. Namely, a regular vortex lattice is observed in the $\mathrm{YBCO}$ and $\mathrm{BSCCO}$ single crystals along with the domain structure caused by weak collective pinning, including that on the oxygen vacancies. The interaction of the magnetic flux irregularity produced by the vortices with the magnetic structure of the $\mathrm{Fe}-\mathrm{As}$ layer [20] could assumingly be responsible for the magnetic structure unusual for the single crystals. However, this assumption contradicts the observations in low magnetic fields where the field in the vortex core is too low to affect the magnetic structure. We can also assume that strong pinning is due to 

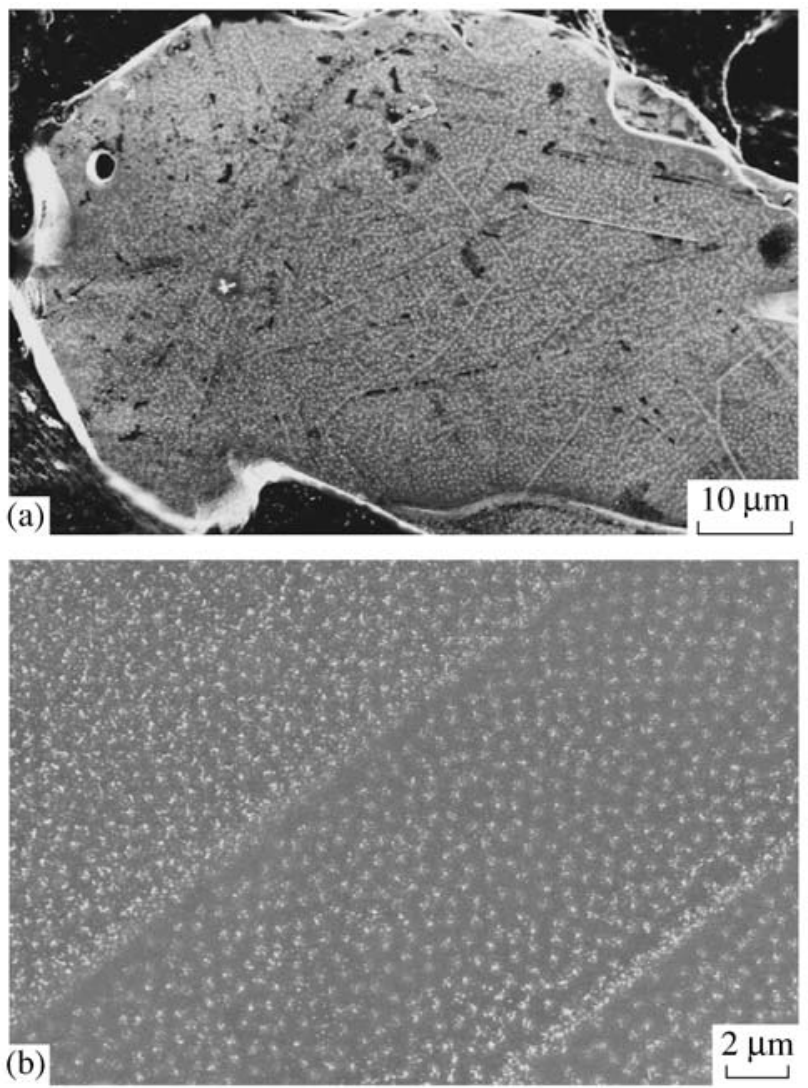

Fig. 2. Vortex structure in a magnetic field of $46 \mathrm{Oe}$ in (a) $\mathrm{SmFeAsO}_{1-x} \mathrm{~F}_{x}$ and (b) $\mathrm{Sr}_{0.85} \mathrm{~K}_{0.15} \mathrm{Fe}_{2} \mathrm{As}_{2}$ single crystals.

the unusual $\pi-\pi$ electron ordering revealed in [21]. To explain the strong pinning in the superconducting pnictide single crystals, one should perform detailed structural (X-ray and electron microscopy) studies, including low-temperature studies in order to reveal the irregularities with sizes of the order of $\xi$ that could be effective pinning centers.

In summary, we have revealed the disordered vortex structure in the single crystals of a new family of hightemperature superconductors based on layered compounds, iron pnictides, which is independent of the crystal structure type, doping, and synthesis methods. This allows us to conclude that the intrinsic mechanism of the vortex pinning of unclear nature exists in the iron pnictide single crystals. Taking into account that the anisotropy in iron pnictides is weaker than that in cuprates, strong pinning should also be expected in polycrystalline samples, and this can be promising for their application.

L.Ya.V., T.M.A., and I.S.V. are grateful to E.Yu. Postnova for assistance in the electron microscopic studies, and to V.N. Zverev and V.A. Gasparov for the measurements of $T_{\mathrm{c}}$. This work was supported by the Russian Foundation for Basic Research (project no. 07-02-00174). The work of the Swiss team was performed within the framework of the MaNEP (Materials with Novel Electronic Properties) project.

\section{REFERENCES}

1. Kenji Ishida, Yusuke Nakai, and Hideo Hosono, J. Phys. Soc. Jpn. 78, 062001 (2009).

2. M. V. Sadovskii, Usp. Fiz. Nauk 178, 1243 (2008) [Phys. Usp. 51, 1243 (2008)].

3. Yu. A. Izyumov and E. Z. Kurmaev, Usp. Fiz. Nauk 178, 1307 (2008) [Phys. Usp. 51, 1307 (2008)].

4. A. L. Ivanovskii, Usp. Fiz. Nauk 178, 1273 (2008) [Phys. Usp. 51, 1273 (2008)].

5. S. Weyeneth, R. Puzniak, N. D. Zhigadlo, et al., J. Supercond. Nov. Magn. 22, 347 (2009).

6. R. Prozorov, N. Ni, M. A. Tanatar, et al., Phys. Rev. B 78, 224506 (2008).

7. N. D. Zhigadlo, S. Katrych, Z. Bukowski, et al., J. Phys.: Condens. Matter 20, 342202 (2008).

8. M. R. Eskildsen, L. Ya. Vinnikov, T. D. Blasius, et al., Phys. Rev. B 79, 100501(R) (2009); Physica C 469, 529 (2009).

9. Yi Yin, M. Zech, T. L. Williams, et al., Phys. Rev. Lett. 102, 097002 (2009).

10. V. V. Shmidt and G. S. Mkrtchyan, Usp. Fiz. Nauk 112 , 460 (1974) [Sov. Phys. Usp. 17, 170 (1974)].

11. A. M. Campbell and J. E. Ivetts, Critical Currents in Superconductors (Taylor and Francis, London, 1972; Mir, Moscow, 1975).

12. J. T. Park, D. S. Inosov, Ch. Niedermayer, et al., Phys. Rev. Lett. 102, 117006 (2009).

13. D. V. Evtushinsky, D. S. Inosov, V. B. Zabolotnyy, et al., Phys. Rev. B 79, 054517 (2009).

14. L. Ya. Vinnikov, I. V. Grigor'eva, and L. A. Gurevich, The Real Structure of High-Tc Superconductors, Ed. by V. Sh. Shekhtman, Springer Ser. Mater. Sci. (Springer, Berlin, Heidelberg, 1993), Vol. 23, p. 89.

15. G. L. Sun, D. L. Sun, M. Konuma, et al., cond-mat. 0901.2728.

16. J. Karpinski, N. D. Zhigadlo, S. Katrych, et al., Physica C 469, 370 (2009).

17. L. Ya. Vinnikov, J. Karpinski, S. M. Kazakov, et al., Phys. Rev. B 67, 092512 (2003).

18. L. Ya. Vinnikov, D. E. Boinagrov, V. N. Zverev, et al., Zh. Eksp. Teor. Fiz. 136, 331 (2009) [JETP 109, 280 (2009)].

19. L. Ya. Vinnikov, L. A. Gurevich, G. A. Emel'chenko, et al., Solid State Commun. 70, 1145 (1989).

20. I. I. Mazin and M. D. Joannes, Nature Phys. 5, 141 (2009).

21. V. B. Zabolotnyy, D. S. Inosov, D. V. Evtushinsky, et al., Nature 457, 569 (2009).

Translated by E. Perova 